\title{
Design and Specific Absorption Rate of 2.6 GHz Rectangular- Shaped Planar Inverted-F Antenna
}

\author{
Nurul Inshirah Mohd Razali, Norhudah Seman, Nur Ilham Aliyaa Ishak \\ Wireless Communication Centre, Faculty of Electrical Engineering, Universiti Teknologi Malaysia, 81310 UTM Johor \\ Bahru, Malaysia
}

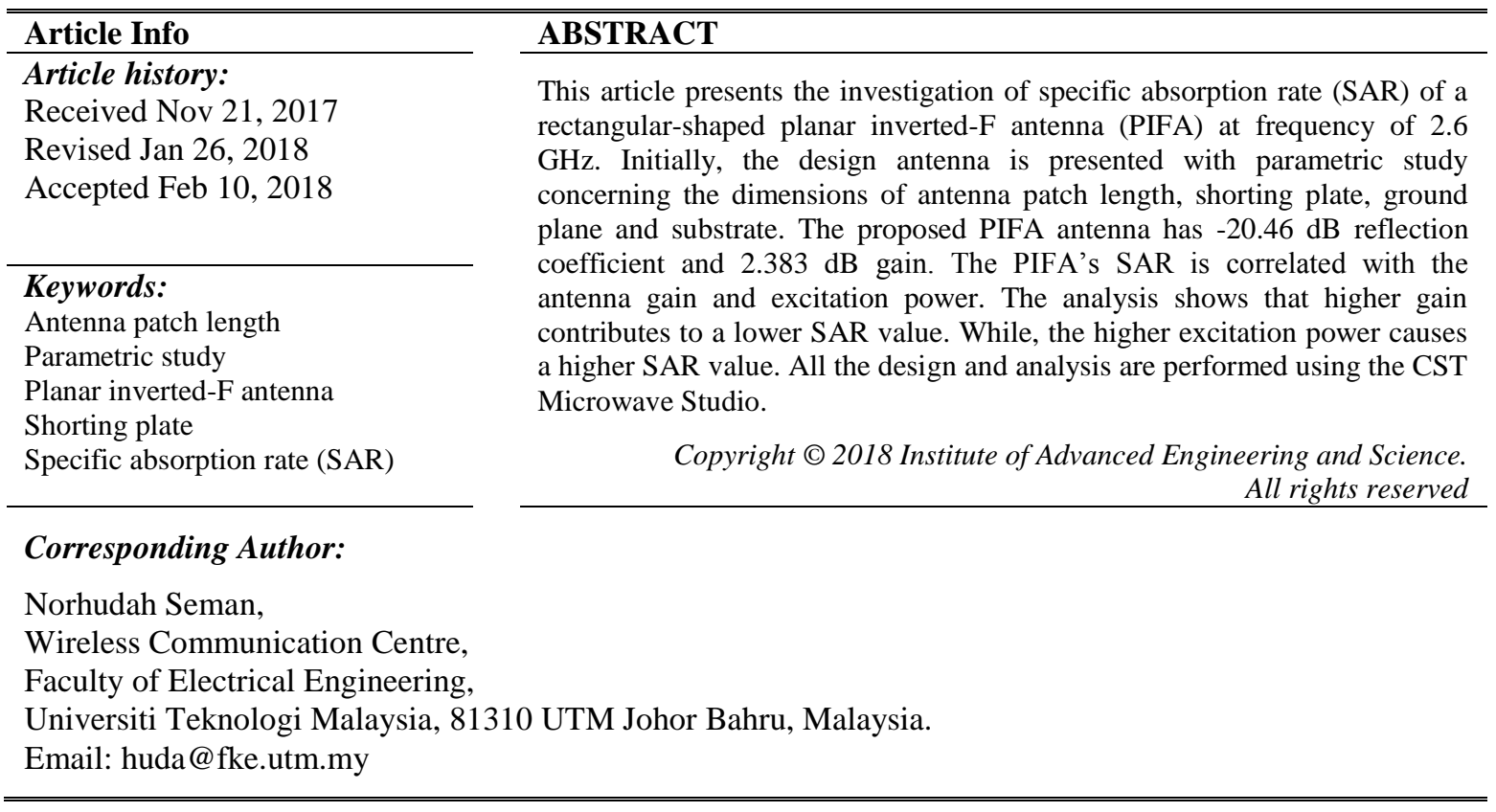

\section{INTRODUCTION}

Wireless devices are essential in connecting numerous users at anytime and anywhere, which require a low profile of antenna. Planar inverted-F antenna (PIFA) can be categorized as one of the lowest profile antenna used widely in a handheld wireless device that has several advantages of simple structure, small size, easy to fabricate and low manufacturing cost [1]-[4]. Furthermore, PIFA gives a smaller size compared to monopole, rod and helix antenna, which lead to easier positioning and alignment between the components [4]. Several researches on PIFA antenna for mobile phone applications are presented in [4] and [5], which ended with large size. PIFA antenna with a total large dimension of $314 \mathrm{x} 157 \mathrm{~mm}$ is presented in [4] implementing a F-shaped slot that realized using RT/Duroid 5880 substrate operated at frequency of 800 $\mathrm{MHz}$. While in [5], the PIFA antenna is designed with a few slots at 3.1-10.6 GHz of operating frequency has a considerable large size of $120 \times 60 \mathrm{~mm}$. The antenna, which embedded in the wireless devices emits the electromagnetic (EM) wave. This EM wave is exposed and absorbed into the human's body. The absorption power rate of the emitted EM wave can be quantified as specific absorption rate (SAR).

SAR is the amount of EM field radiation absorbed by the human body from any wireless devices such as mobile phone. A wireless device's SAR rate is used to estimate the maximum rate of radio frequency (RF) energy absorption by a user's body when using the device [6]. In the United States, the Federal Communications Commission (FCC) sets the SAR exposure limit for the general public to be 1.6 watts per kilogram (1.6 W/kg) [7]. Hence, the mobile device's SAR rate must not exceed $1.6 \mathrm{~W} / \mathrm{kg}$ in order to receive FCC certification and to be sold in the United States. In Europe, the exposure limit is $2.0 \mathrm{~W} / \mathrm{kg}$, averaged over a volume of $10 \mathrm{~g}$ of tissue. The maximum safety limit should not be exceeded to prevent any adverse health effect [8]. SAR value is dependent on the size of the averaging volume, thus the SAR can be expressed by the following theoretical expression [9]: 


$$
\operatorname{SAR}=\frac{1}{V} \int \frac{\sigma(r) l E(r) l^{2}}{\rho(r)} d r
$$

where $\sigma, E, \rho$ and $V$ are the sample of electrical conductivity of body tissue, RMS electric field strength in the tissue, sample density of body tissue and volume of the sample.

Thus, this article proposes a simple and compact rectangular-shaped PIFA antenna operated at LTE operating frequency of $2.6 \mathrm{GHz}$ that has good antenna performance for wireless devices applications. The parametric study is performed to observe the effect of shorting plate, antenna patch length, substrate and ground plane size towards the resonant frequency and antenna gain performance. The investigation of specific absorption rate (SAR) of a PIFA is conducted in correlation to the varied antenna gain and excitation power.

\section{ANTENNA DESIGN AND PARAMETRIC STUDY}

The design of proposed PIFA antenna is shown in Fig. 1. The PIFA antenna consists of a rectangular patch; serve as a radiating element on the top layer. The PIFA antenna is designed based on the following theoretical expression [10]:

$$
\mathrm{Lp}+\mathrm{Wp}=\lambda / 4
$$

where Lp and Wp are the respective width and length of the antenna patch. Meanwhile, $\lambda$ is the wavelength that given by (3):

$$
\lambda=c /\left(f \sqrt{\varepsilon_{e}}\right)
$$

where $c, f$ and $\boldsymbol{\varepsilon}_{e}$ are the speed of light, operating frequency and effective permittivity of the substrate, respectively. The effective permittivity of the substrate, $\boldsymbol{\varepsilon}_{e}$ can be determined from (4) [11]-[13]:

$$
\varepsilon_{e}=\frac{\varepsilon_{r}+1}{2}+\frac{\varepsilon_{r}-1}{2} \frac{1}{\sqrt{1+12 d / \mathrm{Wp}}}
$$

where $d$ and $\mathcal{E}_{r}$ are substrate thickness and relative permittivity. A shorting plate has a dimension of Ws x Ls $\mathrm{x}$ Ts. Where, Ws, Ls and Ts are the respective width, length and thickness of the shorting plate. The shorting plate is located on the left side of the PIFA antenna, which connecting the patch and the ground plane. A coaxial feeding technique is utilized in the proposed design that positioned close to the top left of the PIFA antenna, which offering the best performance. The substrate used for the proposed PIFA antenna is Rogers $\mathrm{RO} 4003 \mathrm{C}$ with a dielectric constant of 3.38, thickness of $0.508 \mathrm{~mm}$, conductor coating of $0.017 \mathrm{~mm}$ and tangent loss of 0.0027 . Several parametric study and optimization are conducted resulting in the optimized antenna patch of $5.88 \mathrm{~mm}(\mathrm{Wp}) \times 13.64 \mathrm{~mm}(\mathrm{Lp})$ and shorting plate size of $0.595 \mathrm{~mm}(\mathrm{Ls})$ x $2 \mathrm{~mm}(\mathrm{Ws}) \times 1$ $\mathrm{mm}$ (Ts). Besides, the size of substrate and ground plane is $55 \mathrm{~mm} \times 55 \mathrm{~mm}$.

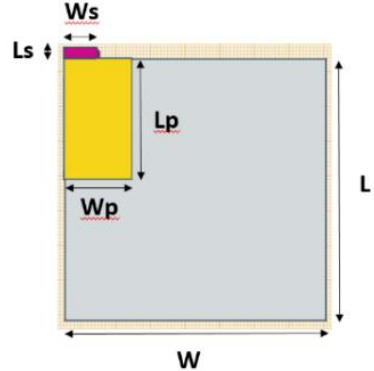

(a)

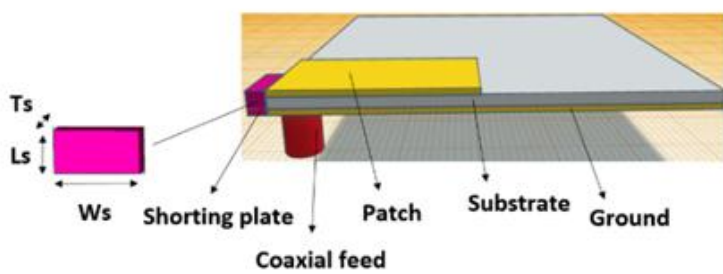

(b)

Figure 1. CST Layout of the Proposed PIFA Antenna: (a) Top View and (b) Perspective View

A parametric study is conducted on several antenna parameters including the size of antenna patch, shorting plate, substrate and ground plane. The parametric study is performed to observe the performance of 
the antenna by focusing on the resonant frequency of $2.6 \mathrm{GHz}$ and the antenna gain. Initially, in designing the antenna, the resonant frequency is shifted from the designated $2.6 \mathrm{GHz}$ to $4.1764 \mathrm{GHz}$. Thus, a few parametric studies have been executed to adjust the resonant frequency towards $2.6 \mathrm{GHz}$ and to achieve the aim of $2 \mathrm{~dB}$ gain. Firstly, the width of shorting plate, Ws is reduced from 12 to $2 \mathrm{~mm}$, while fixing the Ls and Ts dimension to the respective $0.595 \mathrm{~mm}$ and $1 \mathrm{~mm}$. Minimum $2 \mathrm{~mm}$ has taken into deliberation considering fabrication limitation. Fig. 2 (a) shows the proportional relation between the width of shorting plate, Ws and resonant frequency. The best dimension of Ws after the parametric study is $2 \mathrm{~mm}$, which the resonant frequency is shifted to a lower frequency of $3.18 \mathrm{GHz}$. The length of the current-line path increases with decreasing shorting plate width that leads to the decrement of the resonant frequency [14].

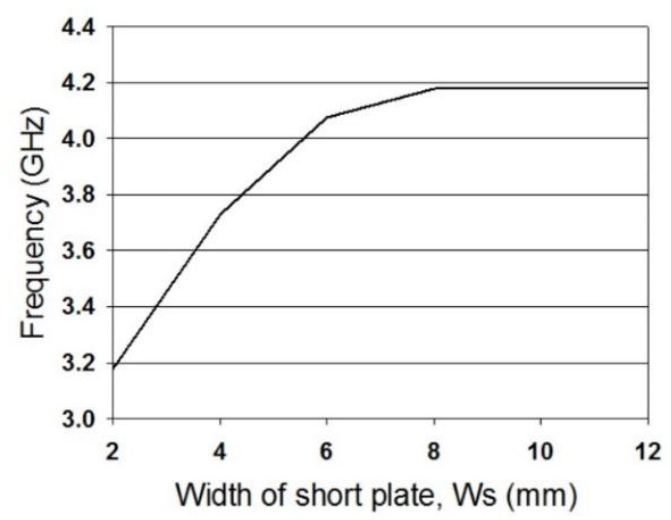

(a)

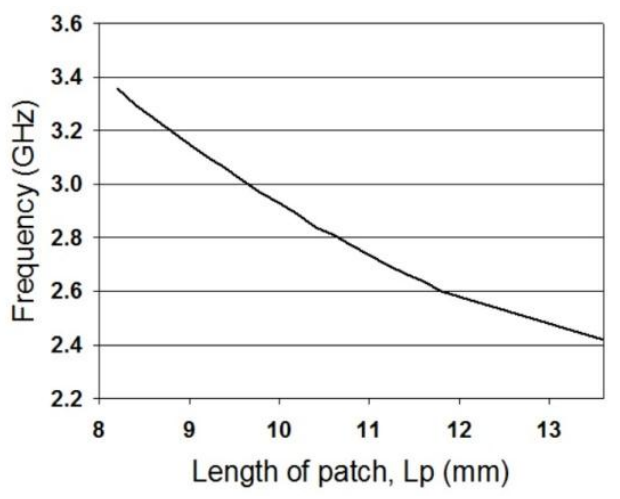

(b)

Figure 2. (a) Resonant Frequency versus Short Plate Width; (b) Resonant Frequency versus Patch Length

Afterward, the patch antenna length, Lp is varied from 8.2 to $14 \mathrm{~mm}$, while its width, Wp is fixed at $5.88 \mathrm{~mm}$ to further decrease the resonant frequency, since the current patch length is increased as presented in Fig. 2 (b). The results depict the increment of antenna patch length has contributed in lowering the resonant frequency. As PIFA is a quarter wavelength antenna, hence by increasing the size of antenna patch, the wavelength increases, which causes the resonance frequency to be decreased as proven from equation (2). The optimal length of antenna patch is $11.8 \mathrm{~mm}$ for the designated resonant frequency of $2.6 \mathrm{GHz}$. However, at this optimal length of the patch antenna, Lp, the antenna has the performance of $1 \mathrm{~dB}$ gain, which lower than the goal of $2 \mathrm{~dB}$. Thus, further parametric study is conducted to obtain the higher antenna gain.

The width, W and length, L of substrate and ground are varied from $30 \mathrm{~mm}$ to $55 \mathrm{~mm}$ to study the effect towards the antenna gain. Fig. 3 shows that $53 \mathrm{~mm} \times 53 \mathrm{~mm}$ of substrate and ground plane dimension has demonstrated the highest gain performance, which is $2.383 \mathrm{~dB}$. However, the resonant frequency of 2.6 $\mathrm{GHz}$ of proposed PIFA antenna is shifted to $2.8 \mathrm{GHz}$. Thus, further optimization is conducted concerning the size of patch length, Lp, while maintaining the width of patch, Wp at $5.88 \mathrm{~mm}$ resulting optimal length of $13.64 \mathrm{~mm}$.

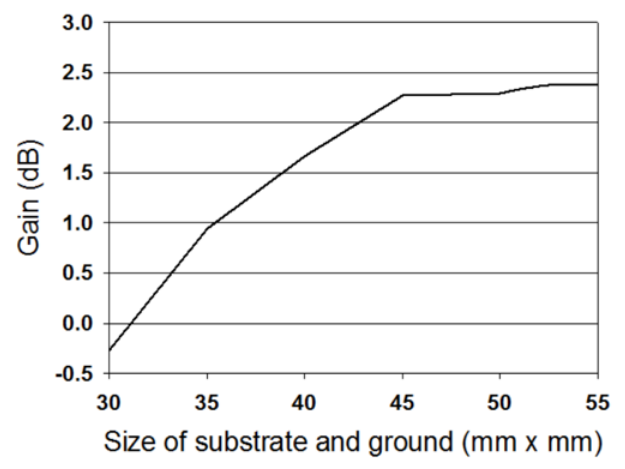

Figure 3. Gain Versus Size of Substrate and Ground 


\section{RESULTS AND DISCUSSIONS}

One of the most important antenna characteristic is the power transmitted by the antenna that can be shown in reflection coefficient, S11 performance at the input port. The results in Fig. 4 indicates that the PIFA antenna is performed at lower than $-10 \mathrm{~dB}$ for both conditions, which are antenna without head phantom and with head phantom, which indicates the proposed PIFA antenna has less than $10 \%$ reflected power. At the designated frequency of $2.6 \mathrm{GHz}$, the $\mathrm{S} 11$ is $-20.46 \mathrm{~dB}$, while the value of VSWR is $1.21 \mathrm{~dB}$ for antenna without head phantom. Fig. 5 shows the radiation pattern of the proposed PIFA antenna with the head phantom and without head phantom. The results of radiation patterns present the nearly omnidirectional beam, which indicating the proposed PIFA antenna is suitable for mobile antennas applications. This result shows that the proposed PIFA antenna is performed well at $2.6 \mathrm{GHz}$ operating frequency.

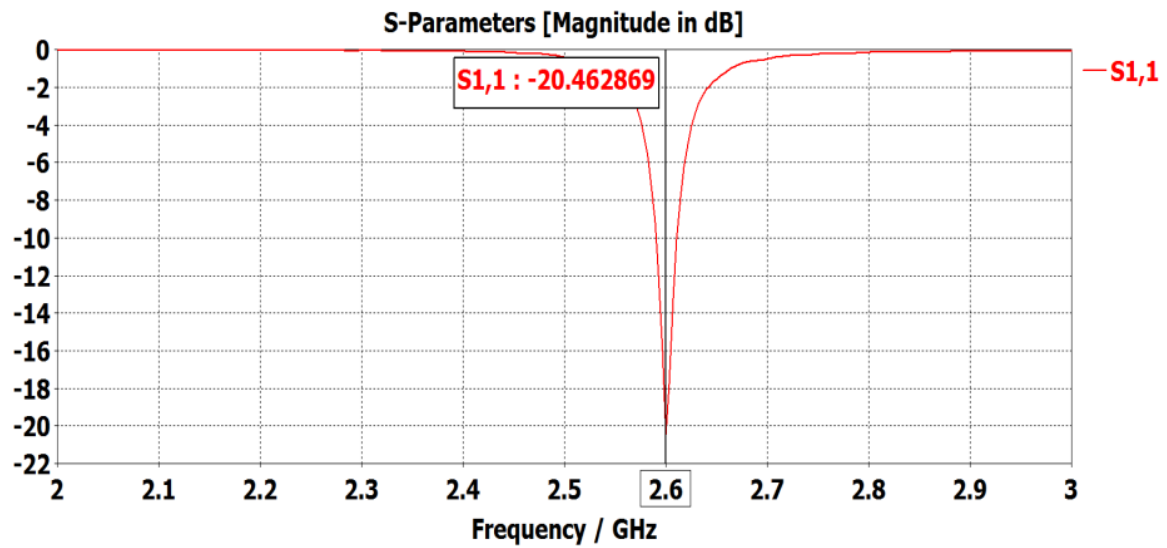

(a)

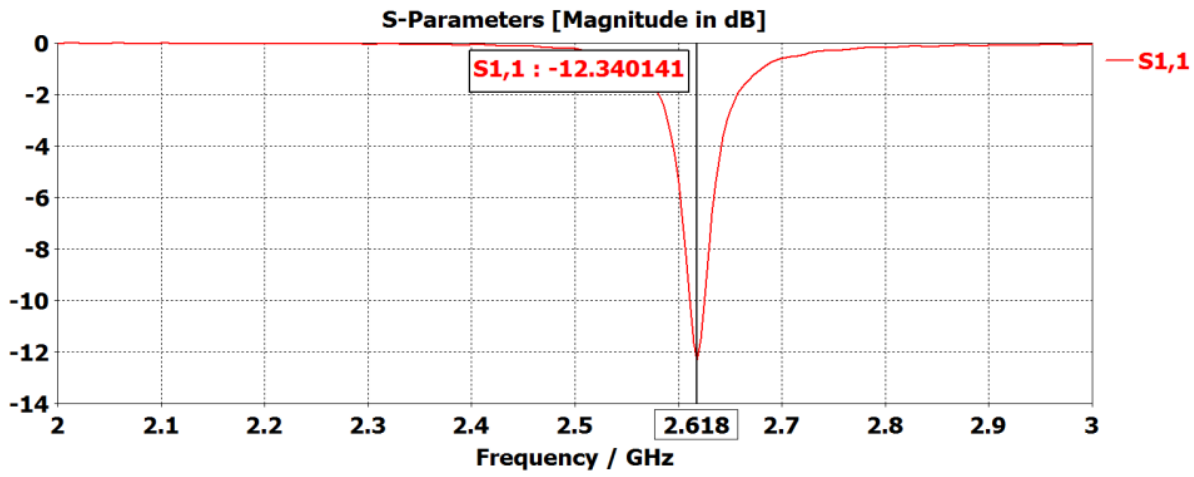

(b)

Figure 4. Reflection Coefficient, S11 of Proposed PIFA Antenna: (a) Without Head Phantom (b) With Head Phantom 


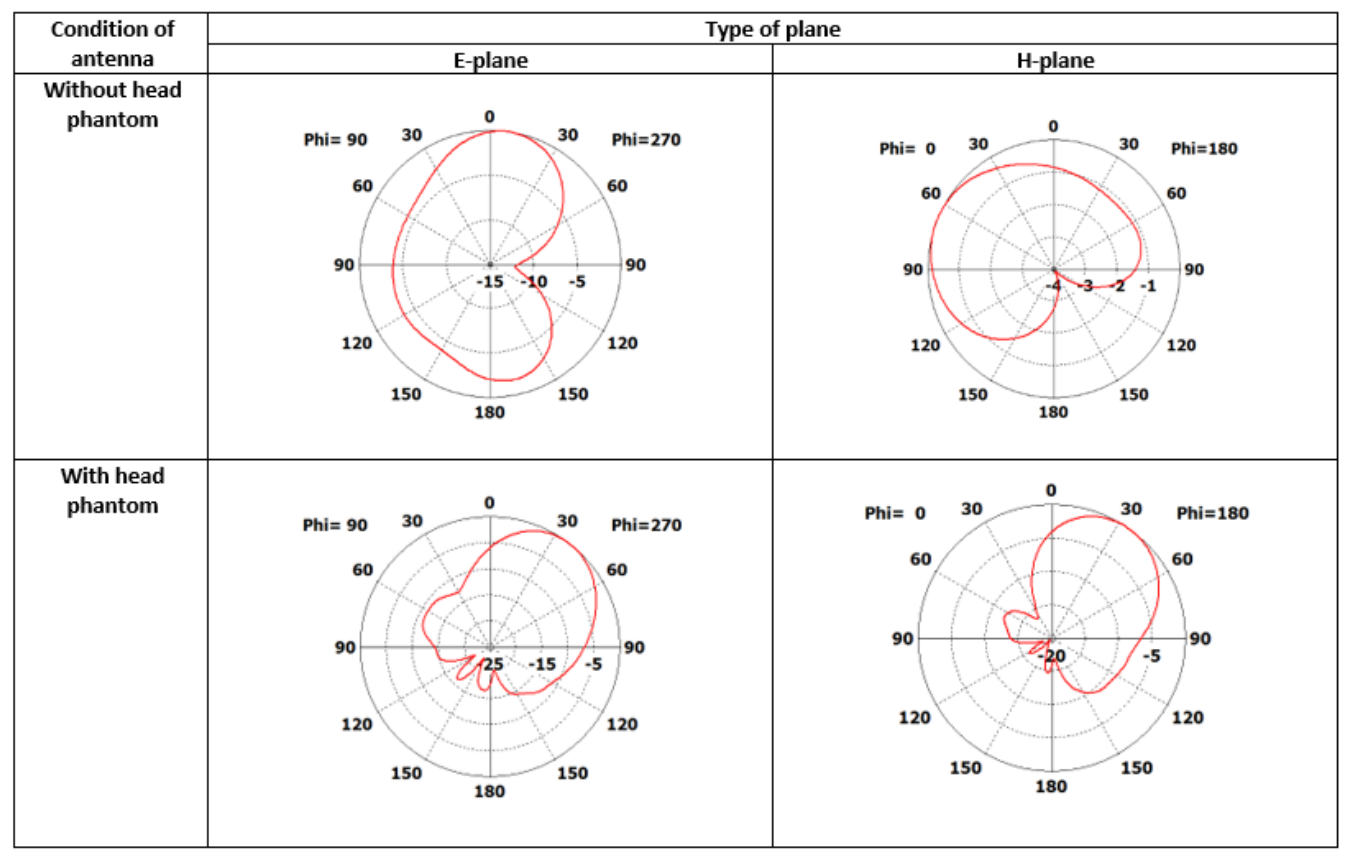

Figure 5. Radiation Pattern of the Proposed PIFA Without and With Head Phantom

Following this, the varied antenna gain and excitation power of PIFA are investigated to observe the effect on SAR performance. The setup for head phantom and PIFA antenna for SAR simulation is shown in Fig. 6. The distance between PIFA and the head model is set to $10 \mathrm{~mm}$.

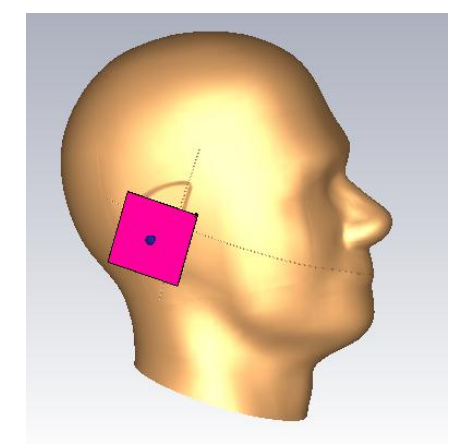

Figure 6. Antenna with Head Phantom

The PIFA antenna gain is influenced by the changing of substrate and ground size from $30 \mathrm{~mm}$ to $50 \mathrm{~mm}$. The change leads to a significant difference in antenna gain of PIFA from a negative value of $0.2674 \mathrm{~dB}$ to the positive value of $2.2970 \mathrm{~dB}$. Each of antenna with different gain value is simulated with a fix averaged excited power of $0.25 \mathrm{~W}$ to investigate the effect on SAR performance. Table 1 shows the simulated SAR with different values of PIFA gains.

Table 1. SAR of the Designed PIFA Compared to Substrate and Ground Size, and Gain

\begin{tabular}{cccc}
\hline $\begin{array}{c}\text { Size of substrate and ground } \\
(\mathrm{mm} \times \mathrm{mm})\end{array}$ & Gain $(\mathrm{dB})$ & $1 \mathrm{~g}$ & $10 \mathrm{~g}$ \\
\hline $30 \times 30$ & -0.2674 & 3.589 & 1.739 \\
$35 \times 35$ & 0.9477 & 3.508 & 1.759 \\
$40 \times 40$ & 1.6666 & 3.086 & 1.591 \\
$45 \times 45$ & 2.2740 & 2.581 & 1.402 \\
$50 \times 50$ & 2.2970 & 1.538 & 0.853 \\
\hline
\end{tabular}


The simulation results show that the SAR performance is closely related to the antenna gain. The higher gain of PIFA antenna resulting the lower value of SAR. When the gain is below than $2.2970 \mathrm{~dB}$, the $\mathrm{SAR}$ value is exceeded the limit, which is above than $1.6 \mathrm{~W} / \mathrm{kg}$. The radiation from the antenna plays an important role in the performance of SAR. When the antenna has a very low gain which means it is not radiating well, it results in a high SAR value

The power excitation also has been verified from $0.1 \mathrm{~W}$ to $0.4 \mathrm{~W}$ to investigate the effect on SAR performance. In this study, antenna with the highest gain, $2.383 \mathrm{~dB}$ is used to run the SAR simulation. The following Table 2 shows the effect of power excitation on the SAR.

Table 2. Variation Excitation Power Towards the SAR Performance of PIFA

\begin{tabular}{ccc}
\hline \multirow{2}{*}{ Power $(\mathrm{W})$} & \multicolumn{2}{c}{$\mathrm{SAR}(\mathrm{W} / \mathrm{kg})$} \\
\cline { 2 - 3 } & $1 \mathrm{~g}$ & $10 \mathrm{~g}$ \\
\hline 0.1 & 0.5316 & 0.3001 \\
0.2 & 1.0630 & 0.6001 \\
0.3 & 1.5950 & 0.9002 \\
0.4 & 2.1260 & 1.2000 \\
\hline
\end{tabular}

Table 2 shows that the increment of excitation power has caused the increment of SAR value. The highest value of excited power can be used is $0.3 \mathrm{~W}$ since it gives the highest SAR value, which is not exceeding the standard limit established, 1.595 for $1 \mathrm{~g}$ of tissue and 0.9002 for $10 \mathrm{~g}$ of tissue. Thus, referring to this analysis, the maximum allowable excitation power for this proposed PIFA is must not higher than 0.3 $\mathrm{W}$ to ensure the SAR value not exceeding the limit of $1.6 \mathrm{~W} / \mathrm{kg}$ and $2.0 \mathrm{~W} / \mathrm{kg}$, for the respective averaged over a volume of $1 \mathrm{~g}$ mass of tisse and $10 \mathrm{~g}$ mass of tissue.

\section{CONCLUSION}

A design of PIFA antenna operated at $2.6 \mathrm{GHz}$ has been proposed. The parametric study is performed on several antenna design parameters to achieve the optimal performance by concerning the operating frequency and the antenna gain. The parametric study results concluded, the size of patch antenna and shorting plate have affected the resonant frequency while, the size of substrate and ground affected the antenna gain performance. The proposed PIFA antenna has been optimized to attain the operating frequency of $2.6 \mathrm{GHz}$ and gain greater than $2 \mathrm{~dB}$. Afterward the investigation on the relation between the gain of PIFA and excitation power used on SAR performance has been conducted. For the gain of PIFA, the simulation results show that the higher gain of antenna has resultsing lower SAR performance. The good radiating performance, which is the high gain of antenna effecting to a good value of SAR (lower SAR). The analysis has shown that $0.3 \mathrm{~W}$ is the maximum allowable excitation power to ensure that the value of SAR is not exceeding the established standard limit.

\section{ACKNOWLEDGEMENTS}

This work was supported by Ministry of Higher-Education Malaysia (MOHE) and Universiti Teknologi Malaysia (UTM) through Prototype Research Grant Scheme (PRGS) [Vote Number of 4L684]; Flagship Grant [Vote Number of 03G41] and HiCoE Grant [Vote Number of 4J212].

\section{REFERENCES}

[1] O. A. Saraereh, M. Jayawadene, P. McEvoy, and J. C. Vardaxoglou, "Simulation and experimental SAR and efficiency study for a dual-band PIFA handset antenna (GSM 900/DCS 1800) at varied distances from a phantom head," Technical Seminar on Antenna Measurements and SAR (AMS), 5-8, 2004.

[2] B. Li, D. Jin, and J. Hong, "Six-band PIFA with low SAR value in mobile phone applications," 2012 International Conference on Microwave and Millimeter Wave Technology (ICMMT), pp. 2-5, 2012.

[3] M. A. Rahman, A. Thakur, and M. O. Goni, "Performance analysis of a planar inverted-F antenna (PIFA) including human interaction," 2013 Int. Conf. Informatics, Electron. Vision, ICIEV 2013, no. 1, 2013.

[4] N. Kumar, A. Thakur, and J. Sharma, "Study of planar inverted-F antenna (PIFA) for mobile devices," Int. J. Electron. Commun. Technol., vol. 7109, pp. 83-85, 2013.

[5] S. Jegadeesan, Z. Mansouri, A. Veeramani, and F. B. Zarrabi, "Ultra wideband PIFA antenna with supporting GSM and WiMAX for mobile phone applications," Int. Conf. Adv. Comput. Commun. Technol. ACCT, vol. 2015-April, pp. 15-20, 2015. 
[6] R. Yahya, M. R. Kamarudin, and N. Seman, "New Wideband Textile Antenna for SAR Investigation in Head Microwave Imaging," Conf. Proc. - 2014 IEEE MTT-S Int. Microw. Work. Ser. RF Wirel. Technol. Biomed. Healthc. Appl. IMWS-Bio 2014, 2015.

[7] Federal Communication Commission, "Evaluating Compliance with FCC Guidelines for Human Exposure to Radiofrequency Electromagnetic Fields Supplement B," vol. 65.

[8] N. A. Elias, et al. "SAR levels for irradiation by a crumpled $900 \mathrm{MHz}$ flexible diamond dipole." International Journal of Electrical and Computer Engineering (IJECE) $7.3,2017$.

[9] IEEE Standard for Safety Levels with Respect to Human Exposure to Radio Frequency Electromagnetic Fields, 3 kHz to $300 \mathrm{GHz}$, vol. 2005, no. April. 2006.

[10] H. T. Chattha, Y. Huang, X. Zhu, and Y. Lu, "An empirical equation for predicting the resonant frequency of planar inverted-F antennas," IEEE Antennas Wirel. Propag. Lett., vol. 8, pp. 856-860, 2009.

[11] D. M. Pozar. Microwave engineering. John Wiley \& Sons, 2009.

[12] S. Elajoumi, A. Tajmouati, A. Errkik, A. Sanchez and M. Latrach. "Microstrip rectangular monopole antennas with defected ground for UWB applications," International Journal of Electrical and Computer Engineering (IJECE), vol. 7, no. 4, pp. 2027-35, 2017.

[13] B. Garg, D. Saleem. "Innovative double H metamaterial structure for amelioration in patch antenna parameters," Bulletin of Electrical Engineering and Informatics 2013 (BEEI); 2(4): 278-285.

[14] H. Chen, Y. Lin, P. Cheng, H. Lin, C. T. P. Song, and P. S. Hall, "Parametric study on the characteristics of planar inverted-F antenna," IEE Proceedings - Microwaves, Antennas and Propagation, vol. 152, no. 6, pp. 534$538,2005$.

\section{BIOGRAPHIES OF AUTHORS}

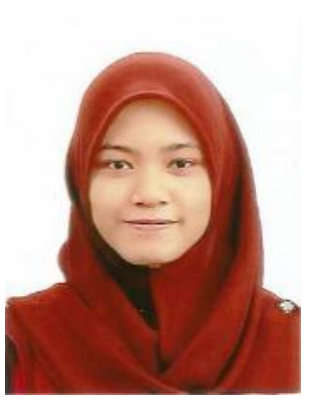

Nurul Inshirah Mohd Razali is a student of Master of Philosophy in Electrical Engineering from Wireless Communication Centre, Universiti Teknologi Malaysia. Inshirah has completed her degree in Electrical-Electronic Engineering from Universiti Teknologi Malaysia. Her current area of interest is in Specific Absorption Rate (SAR) investigation on PIFA antenna.

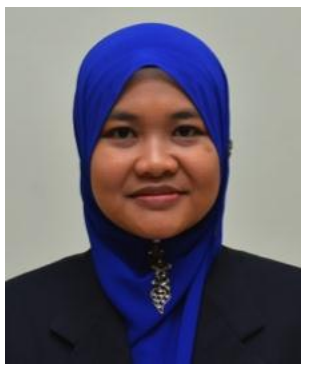

Norhudah Seman received the B. Eng. in Electrical Engineering (Telecommunications) degree from the Universiti Teknologi Malaysia, Johor, Malaysia, in 2003 and M. Eng. degree in RF/Microwave Communications from The University of Queensland, Brisbane, St. Lucia, Qld., Australia, in 2005. In September 2009, she completed her Ph.D. degree at The University of Queensland. In 2003, she was an Engineer with Motorola Technology, Penang, Malaysia, where she was involved with the RF and microwave components design and testing. Currently, she is a Associcate Professor in Wireless Communication Centre (WCC), Universiti Teknologi Malaysia. Her research interests concern the design of microwave circuits for biomedical and industrial applications, specific absorption rate (SAR), UWB technologies, and mobile communications.

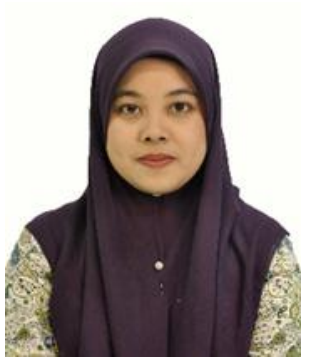

Nur Ilham Aliyaa binti Ishak was born on 9th January 1989. She received the B.S degree in Electrical and Electronic Engineering from Universiti Sains Malaysia, Pulau Pinang, in 2012, whereas M.Sc in Physics from Universiti Malaysia Terengganu in 2015. Her current research interest include multiple antennas for MIMO applications, electromagnetic interaction with human and EM field absorption (SAR). 